Canad. Math. Bull. Vol. 20 (1), 1978

\title{
A SUM OF RECIPROCALS OF LEAST COMMON MULTIPLES
}

\author{
D. BORWEIN
}

The purpose of this note is to prove the following theorem conjectured by $\mathrm{P}$. Erdös.

THEOREM. Let $a_{0}, a_{1}, \ldots, a_{k}$ be integers satisfying $1 \leq a_{0}<a_{1}<\cdots<a_{k}$, and let $\left[a_{i-1}, a_{i}\right]$ denote the least common multiple of $a_{i-1}$ and $a_{i}$. Then

$$
\frac{1}{\left[a_{0}, a_{1}\right]}+\frac{1}{\left[a_{1}, a_{2}\right]}+\cdots+\frac{1}{\left[a_{k-1}, a_{k}\right]} \leq 1-\frac{1}{2^{k}}
$$

with equality occurring if and only if $a_{i}=2^{i}$ for $1 \leq i \leq k$.

Proof. For $i=1,2, \ldots, k$, let $c_{i}=\left[a_{i-1}, a_{i}\right]$, and let

$$
s_{i}=\frac{1}{c_{1}}+\frac{1}{c_{2}}+\cdots+\frac{1}{c_{i}} .
$$

Then $c_{i}=u_{i} a_{i-1}=v_{i} a_{i}$ where $u_{i}>v_{i} \geq 1$. Hence

$$
\frac{1}{c_{i}} \leq \frac{1}{a_{i}},
$$

and, since $c_{i}^{-1} \leq\left(u_{i}-v_{i}\right) c_{i}^{-1}$,

$$
\frac{1}{c_{i}} \leq \frac{1}{a_{i-1}}-\frac{1}{a_{i}} .
$$

It follows from (3) that

$$
s_{i} \leq \frac{1}{a_{0}}-\frac{1}{a_{i}}
$$

To establish (1) we consider three cases which exhaust all possible conditions on the integers $a_{0}, a_{1}, \ldots, a_{k}$.

CASE 1. $a_{k} \leq 2^{k}$. Then, by (4),

$$
s_{k} \leq 1-\frac{1}{a_{k}} \leq 1-\frac{1}{2^{k}} .
$$

Received by the editors December 15, 1976. 
CASE 2. $a_{i}>2^{i}$ for $1 \leq i \leq k$. Then, by (2),

$$
s_{k} \leq \frac{1}{a_{1}}+\frac{1}{a_{2}}+\cdots+\frac{1}{a_{k}}<\frac{1}{2}+\frac{1}{2^{2}}+\cdots+\frac{1}{2^{k}}=1-\frac{1}{2^{k}} .
$$

CASE 3. $a_{j} \leq 2^{j}$ for some positive integer $j<k$, and $a_{i}>2^{i}$ for $j+1 \leq i \leq k$. Then, by (2) and (4),

$$
s_{k}=s_{j}+\frac{1}{c_{j+1}}+\cdots+\frac{1}{c_{k}}<1-\frac{1}{2^{j}}+\frac{1}{2^{j+1}}+\cdots+\frac{1}{2^{k}}=1-\frac{1}{2^{k}} .
$$

Thus (1) holds in all three cases. Further, it is immediate that equality occurs in (1) when $a_{i}=2^{i}$ for $1 \leq i \leq k$.

Suppose next that

$$
s_{k}=1-\frac{1}{2^{k}}
$$

Then, by (4), we have $1-2^{-k} \leq 1-a_{k}^{-1}$ so that $a_{k} \geq 2^{k}$; and we cannot have $a_{k}>2^{k}$ for Case 2 and Case 3 show that this would lead to $s_{k}<1-2^{-k}$. Hence

$$
a_{k}=2^{k} \text {. }
$$

If $k=1$ there is nothing further to prove. For $k>1$, we have, by (1) with $k-1$ in place of $k$, and (2), that

$$
1-\frac{1}{2^{k-1}} \geq s_{k-1}=s_{k}-\frac{1}{c_{k}} \geq 1-\frac{1}{2^{k}}-\frac{1}{2^{k}}=1-\frac{1}{2^{k-1}} .
$$

Hence

$$
s_{k-1}=1-\frac{1}{2^{k-1}}
$$

and repetition yields the desired conclusion that

$$
a_{i}=2^{i} \text { for } 1 \leq i \leq k \text {. }
$$

The University of Western Ontario

London, Ontario, Canada N6A 5B9 